\title{
Evaluation of Input Method by Myoelectric Potential Sensor for Eye-Tracking System
}

\author{
Naoto Mukai* and Hisano Goto \\ Department of Culture-Information Studies, \\ School of Culture-Information Studeis, \\ Sugiyama Jogakuen University \\ 17-3, Hoshigaoka-motomachi, Chikusa-ku, \\ Nagoya, Aichi, Japan
}

*Corresponding Author: nmukai@sugiyama-u.ac.jp

\begin{abstract}
Persons with a disability like ALS or SMA patients have difficulty in moving their bodies. One way to communicate with others for them is the eye-tracking system. However, some of them cannot keep their line of sight and fail their input operations by eye-tracking. Therefore, we developed an input method by myoelectric potential for eye-tracking users. It works like a mouse click and assists the user's operation when they choose an option on the display while using eye-tracking. In this paper, we compare with three input methods: eye-tracking with time, eye-tracking with a mouse click, and eye-tracking with a myoelectric potential. Our experimental result indicated that myoelectric potential based input method is competitive with other methods.
\end{abstract}

Keywords: Eye-Tracking, Myoelectric Potential, and Disability person.

\section{Introduction}

According to the 2018 annual report on government measures for persons with disabilities ${ }^{1}$, there are about 4.3 million disabled persons in Japan, and the number of the peolpe is increasing yearly. In order to encourage social involvement for the people, various welfare devices are developed. Especially, communication devices are the key issue for the problem. One of the useful communication devices is Eye-Tracking System, e.g., Tobii $\mathrm{Pro}^{2}$. The

\footnotetext{
1 https://www8.cao.go.jp/shougai/whitepaper/h30hakusho/zenbun/

2 https://www.tobiipro.com/
}

eye-tracking system detects the movement of people's eyes and estimates the focus points on the display. Thus, persons with a disability like ALS or SMA patients, who have difficulty in moving their bodies, need the eye-tracking device for their communication. However, some of them cannot keep their line of sight and often fail their input operations by eye-tracking.

Osugi et. al. proposed a developing system of course materials for schools by using eye-tracking for disabled children [1]. The system has the potential to reduce the input time compared with the traditional switch systems, e.g., mouse click. Kitamura et al. improved the input time for ALS patients by using Brain Computer Interface (BCI) [2]. The BCI is a lower physical stress method than eye-tracking. However, BCI has a difficult task of convenience, i.e., electric poles must be put on their bodies.

In this paper, we try to reduce the input time by eye-tracking by introducing an input method based on Myoelectric Potential. The myoelectric potential is generated by the myoelectric activity of the human body. The sensor of myoelectric potential is more convenient than the $\mathrm{BCI}$ because the size of the sensor is smaller than the BCI devices. The myoelectrical potential is already used for various purposes. For example, Hyuga et. al. proposed a lightweight robot arm which can be controlled by the myoelectrical potential [3]. Our input method by using myoelectric potential works like a mouse click and assists the user's operation when they choose an option on the display while using eye-tracking. In order to evaluate the 
usefulness of our input method, we compare with three input methods: eye-tracking with time, eye-tracking with a mouse click, and eye-tracking with a myoelectric potential sensor.

The structure of this paper as follows. Section 2 shows the game we developed for evaluation and the detail of the three input methods. Section 3 reports the experimental results and discusses the availability of the input method. Section 4 concludes this paper and offers our future works.

\section{Input Methods for Eye-Tracking}

This section describes the detail of three input methods for eye-tracking we compared in our experiment.

\subsection{Either-or Decision Game}

In order to evaluate input methods, we developed a simple either-or decision game. Fig. 1 shows a screenshot of the game. As shown in the figure, the game shows two numbers. A user must choose a bigger number from the two within the given time (10 seconds). The game is very easy for anyone except for small children, but rushing often makes fails of their choice.

\subsection{Input Methods}

Here, we explain the detail of input methods for eye-tracking: eye-tracking with time, eye-tracking with a mouse click, and eye-tracking with a myoelectric potential sensor. We adopted a major eye-tracking device "Eye Tracker 4C for PC Gaming" developed by Tobii.

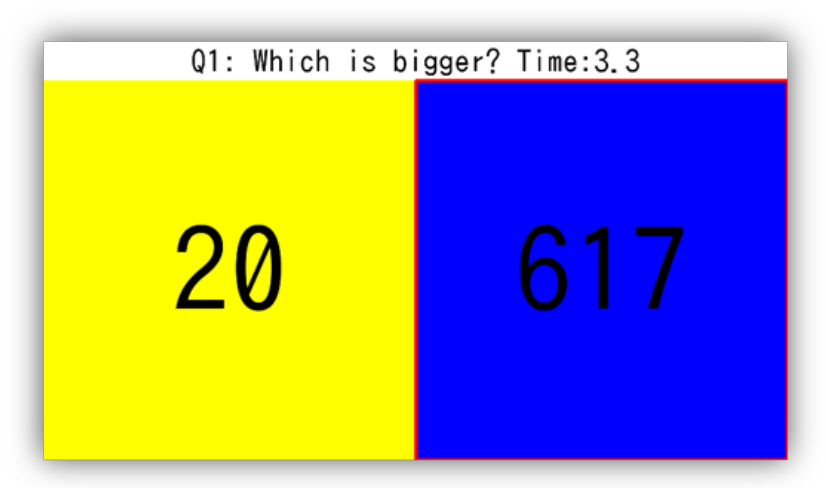

Fig. 1. Screenshot of Either-or Decision Game

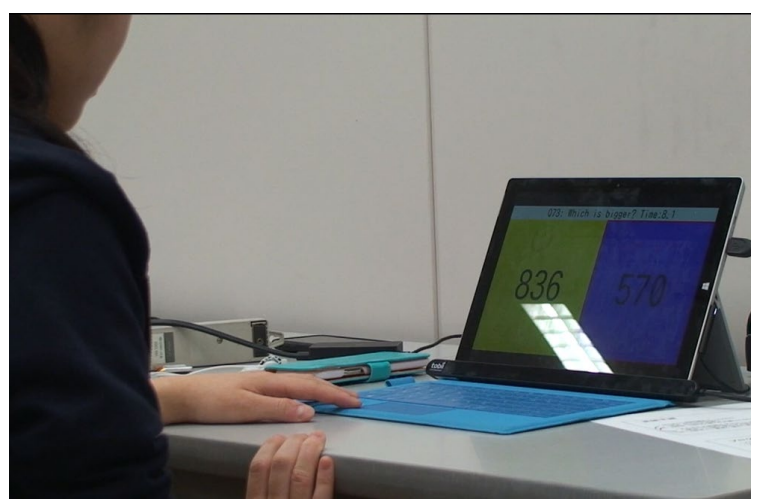

Fig. 2. Eye-Tracking Device

The first input method is "eye-tracking with time". The trigger of this input method is elapsed time. This means, if the time to gaze the number is longer than a threshold (1.5 seconds), the number is chosen by the user. This method is the most convenient because it does not need other sensor devices. However, gazing the same point for a constant time is tired task and look away often cause the failure of choosing the number. Moreover, some of the disabled persons cannot keep their line of sight. Fig. 2 shows a user who plays the game by the eye-tracking device.

The second input method is "eye-tracking with a mouse click". The trigger of this input method is a mouse click. This means that the number gazed by the user is chosen at the moment when the user clicks the button of the mouse. This method is a natural way for the able-bodied person and a fast and accurate way compared to other methods. Note that the position of a mouse cursor is not applied to choose the bigger number.

The third input method is "eye-tracking with a myoelectric potential sensor". The trigger of this input method is the change of myoelectric potential. This means that the number gazed by the user is chosen at the moment when the myoelectric potential becomes higher than the threshold value. Here, we set the threshold as the average of the potential $+100(\mathrm{mv})$. Fig. 3 shows the sensors of the myoelectric potential. The value of the sensor is sent to a

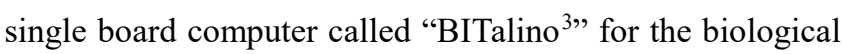
signal.

\footnotetext{
3 http://bitalino.com/en/
} 


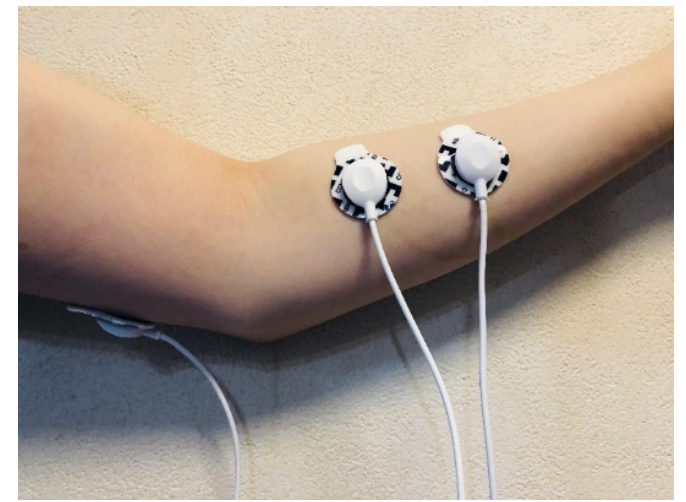

Fig. 3. Sensor of Myoelectric Potential

\section{Experiments}

This section reports the comparison results with above three input methods.

\subsection{Experimental Outline}

Here, we show the outline of our experiment. The objective of this experiment is the comparison with above three input methods. The total number of subjects is 16 , and all of them are able-bodied students of Sugiyama Jogakuen University. Each subject plays the either-or decision game and gives an answer to 100 questions in each input method. We take the average of response time (seconds) and the proportion of correct answers. Moreover, we applied the paired t-test to all combinations of the input methods.

\subsection{Experimental Results}

Fig.4 shows the proportion of correct answers. We found that the most accurate method is eye-tracking with time. It seems that the wrong answers can be reduced because our game just only shows two numbers and the display size of the number is big. On the other hand, eye-tracking with mouse click is the worst result. There is significantly different between the two methods ( $p$-value is 0.018). It is obvious that a mouse click is fast way for able-bodied persons, but they feel rushed and made careless mistakes. Eye-tracking with myoelectric potential is the middle score among them. There is no significantly different between the eye-tracking with myoelectric potential and others.

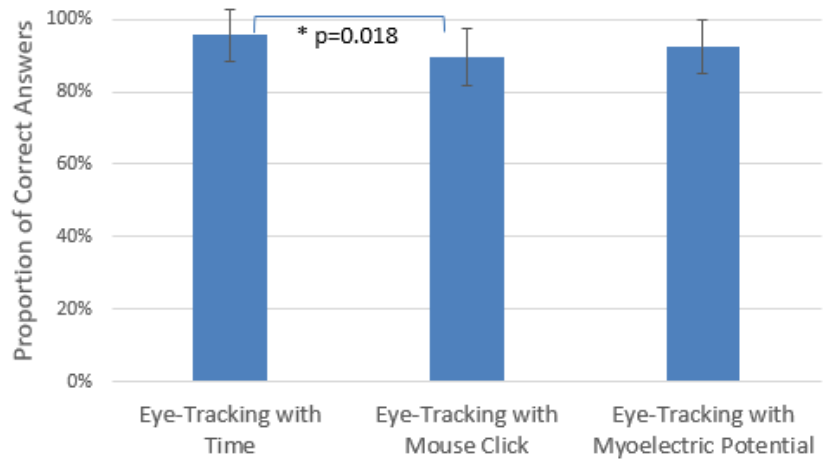

Fig. 4. Proportion of Correct Answers

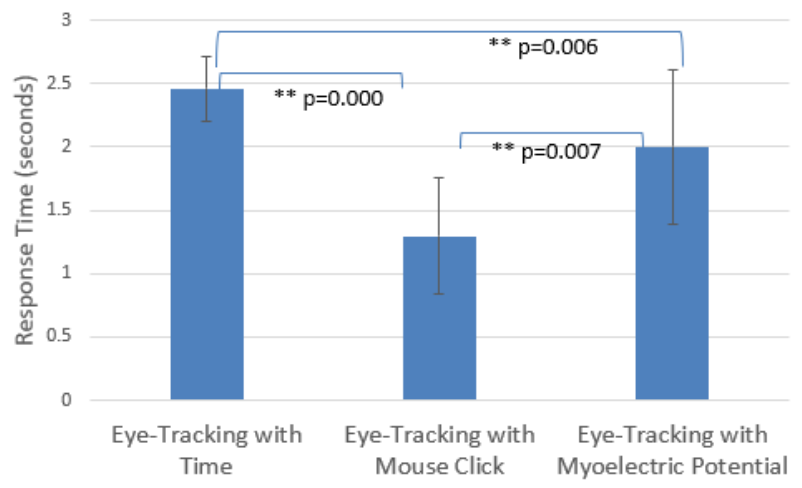

Fig. 5. Response Time (seconds)

Fig.5 shows the response time (seconds). We found that the fastest method is eye-tracking with a mouse click as expected. The next fast method is eye-tracking with myoelectric potential. It has significantly different to eye-tracking with time ( $\mathrm{p}$-value is 0.006 ). This fact means that the input method using myoelectric potential can improve the input time of eye-tracking. However, in this case, we set the threshold at 1.5 seconds. If this threshold can be short, the difference between the two may be reduced. It also may depend on user's habituation.

After the experiment, we asked the subjects about the difficulty of input methods. Fig. 6, 7, and 8 are the results about the difficulty. The results indicated that eye-tracking with a mouse click is the easiest way for able-bodied persons as expected. Moreover, we found that eye-tracking with myoelectric potential is not easy way compared to other methods. Some of the subjects said, "the myoelectric potential sensor was not activated even though I put some muscle". This fact indicated that the adjustment of the threshold is sensitive and difficult. 


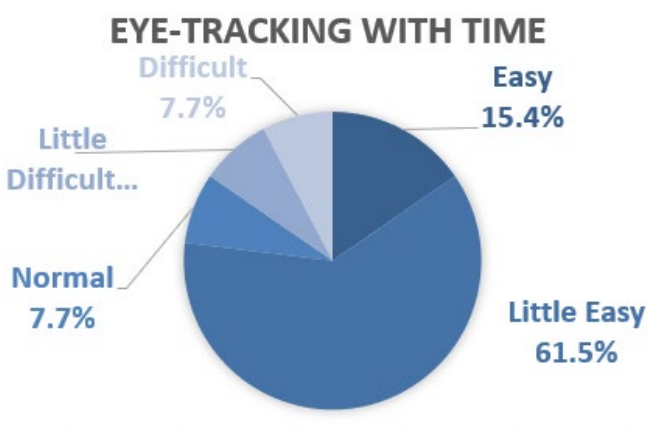

Fig. 6. Difficulty of Eye-Tracking with Time

\section{EYE-TRACKING WITH MOUSE CLICK}

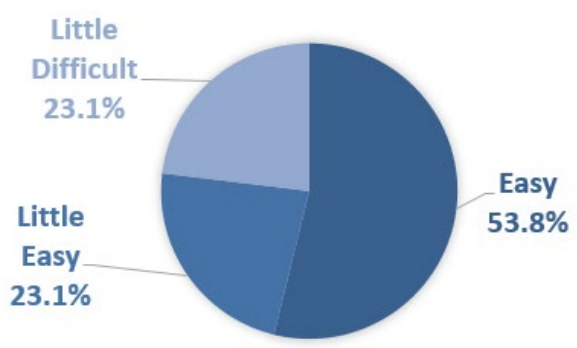

Fig. 7. Difficulty of Eye-Tracking with Mouse Click

\section{EYE-TRACKING WITH MYOELECTRIC POTENTIAL}

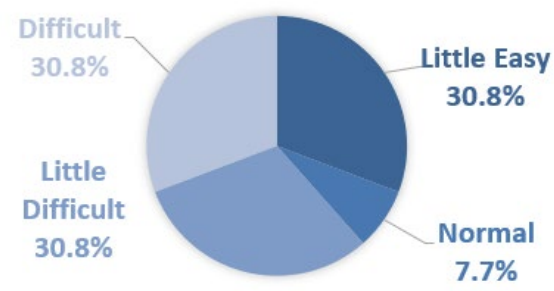

Fig. 8. Difficulty of Eye-Tracking with Myoelectric Potential

\section{Conclusions}

This work focused on eye-tracking users, especially persons with a disability like ALS or SMA patients. They have difficulty in moving their bodies and need the eye-tracking device for their communications. We developed an input method based on the myoelectric potential to reduce input time while using eye-tracking. The comparison results indicated that the input method based on myoelectric potential performed competitive results with other methods. However, the method is difficult for users. This is considered because the setting of the threshold is very sensitive.

In order to overcome the problem, we must develop an automatic adjustment system for the threshold of myoelectric potential. We think some machine learning techniques can be applied to the purpose. Moreover, we need to evaluate our input method by persons with a disability like ALS or SMA patients.

\section{Acknowledgment}

We would like to thank our great adviser, Mr. Hisamu Sato. This work is supported by Tateishi Science and Technology Foundation.

\section{References}

(1) Nariki Osugi and Hideo Kobayashi: “A Proposal for Development of Easy-to-Modify Teaching Materials Incorporated with Eye-Tracking and Signal Output Devices for the Learning of the Children with Severe and Multiple Disabilities", Journal of Japan Society for educational technology, Vol. 40, pp.149-152, 2017

(2) Shota Kitamura and Hisaya Tanaka: "Improvement of Input Time for Communication Aid on ALS Patients", IEEJ Transactions on Electronics, Information and Systems, Vol.138, No.6, pp.641-647, 2018

(3) Hyuga Fuki and Toshiya Arakawa, "Design and Evaluation of Low Cost Lightweight Robot Arm Using Myoelectric Potential Sensor", 